\title{
MULTI OBJECTIVE SUPPLY CHAIN NETWORK RECONFIGURATION UNDER DISRUPTIONS
}

\author{
Varthini Rajagopal $^{1}$, S. Prasanna Venkatesan ${ }^{2}$ and Rahul Agrawal ${ }^{3}$ \\ ${ }^{I}$ Dept. of Production Engg, National Institute of Technology, Tiruchirapalli, India \\ ${ }^{21}$ Dept. of Production Engg, National Institute of Technology, Tiruchirapalli, India \\ ${ }^{3}$ Dept. of Production Engg, National Institute of Technology, Tiruchirapalli, India
}

\begin{abstract}
Disruption in today's complex and global supply chain network ( $\mathrm{SCN}$ ) results in a negative impact on business performance. Companies try to manage disruptions by shifting the production/sourcing to undisrupted facilities, expanding the capacity at selected facilities, re-routing transportation and outsourcing the unmet demands. Models and methods to find a cost effective SCN reconfiguration to deal with disruption need attention. In this work, a SCN reconfiguration in a dynamic planning horizon under facility disruption is modelled mathematically with the objectives of minimizing the expected total cost and delivery time. The augmented $\varepsilon$-constraint method is proposed as a solution approach to obtain a set of Pareto optimal solutions. Numerical illustration with disruption scenarios is presented and our results show that facility with minimum distance to most customers serve majority of total customer demand. During disruption onset period, unmet demands are shifted to least utilised facility. During recovery periods, the facility with minimum distance to most customers is expanded to satisfy unmet demands. More capacity expansion occurred in minimum cost solution than in minimum time solution as more number of facilities are opened in latter than in former. The capacity utilization of facilities in minimum cost solutions are higher compared to that of minimum time solution.
\end{abstract}

Keywords: Supply chain network reconfiguration, facility disruption, capacity expansion, p-robust, multi-objective, multi-period

\section{Introduction}

Modern supply chains are cost oriented and many fail to consider the need for investing in disruption recovery management as they decrease the net profit. Past research in supply chain disruptions (refer Tang 2006 and Snyder et al., 2016) have focussed mainly on proactive mitigation approaches. It is difficult to justify the investments made in proactive disruption management from supply chain manager's perspective (Ghadge et al., 2015 and Dani, 2008). Reconfiguring the supply chain network (SCN) by managing with the available resources and restoring to normal working condition is a reactive disruption mitigation strategy (Blackhurst et al., 2005; Tang, 2006 and Baymout, 2014). Supply chain network reconfiguration (SCNR) under disruption includes strategies such as shifting the production to undisrupted facilities, capacity expansion at selected facilities, opening new facilities, inventory allocation, transportation rerouting and outsourcing unmet demand(Wilhelm et al., 2013; Thanh et al., 2008 and
Melo et al., 2006). Recent reviews on facility location dynamics suggest that network reconfiguration under facility disruption risk as a high scope problem (Seyedhosseini et al., 2016 and Arabani \& Farahani, 2012).Past SCNR models deal mainly with operational risks such as demand, supply, commodity price fluctuation, exchange rate and import tariff risks (Cortinhal et al., 2015; Pimentel et al., 2013; Melo et al., 2012, 2006 and Thanh et al., 2011). However, in practise the loss due to low probable catastrophic disruptions are significant as compared to repetitive operational risks. Ivanov et al., (2016) have presented a multi-stage SC re-planning model under facility and transportation disruptions considering gradual capacity recovery and disruption duration. The optimal proactive SC structure and recovery policies (such as opening new back-up suppliers, depots and transportation channels/modes and use of inventory and capacity buffers) against disruptions are determined using System dynamics and linear 
programming. Kristianto et al., (2014) developed a two stage stochastic program for designing a reconfigurable supply chain network under disruptions by optimal inventory allocation and transportation routing. Klibi \& Martel, (2013) incorporated operational response and structural adaptation decisions (such as additional expediting, backorder and overtime recourse decisions) in each SC planning period and developed a multi-criteria design evaluation approach to select the most effective and robust SCND among candidate solutions. Summarizing from literature, clearly SCNR models considering facility disruption received limited attention (Ivanov et al., 2016, Kristianto et al., 2014 and Klibi \& Martel, 2013). Motivated by this clear research gap, we develop a multi-objective multi-period SCNR model under facility disruptions. In particular, our model answers the following issues:

- Which facilities to open at the beginning of the planning period such that the expected cost of reconfiguration is restricted by a user- defined threshold i.e. p-robustness criteria(Snyder and Daskin, 2006 and Peng et al., 2011)

- How the material flow in SC should be directed in each planning period in the event of facility disruptions such that demand requirements are satisfied while minimizing cost and delivery time?

- When and which undisrupted facilities should be selected for capacity expansion?

- When and which customer's unmet demand should be outsourced?

Following the work of Peng et al., (2011),a p-robust mixed integer linear programming (MIP) model is developed with the objectives of minimizing the expected cost of reconfiguration and delivery time. The proposed model differs from the existing literature on SCNR in some directions. First, the existing models typically discuss proactive strategies such as backup suppliers, backup depots and transportation channels/ modes, inventory and capacity buffers and facility fortification against disruption. Whereas our model addresses recovery strategies like shifting production/ sourcing to undisrupted facilities, expanding capacity of undisrupted facilities and outsourcing demand to external facility. Second, our model incorporates a set of reconfiguration planning periods among the planning horizon following an onset of disruption during which the material flow is reassigned and SC structural adaptation decisions are made. To the best of our knowledge except the work of Klibi \& Martel, (2013), all the above cited literature on SCNR models assume that SC structural adaptation decisions can be made anytime within the planning horizon to tackle the operational and disruption risks which are highly impractical. Third, most of the above mentioned reconfiguration models are single objective dealing either with SC cost minimization or profit maximization. Whereas our multi-objective recovery model finds a trade-off between minimising the expected cost of reconfiguration after the onset of a facility disruption while meeting the customer service level. Furthermore, SCNR models in literature use expected measures like expected cost, expected service level, etc. and mean-variance measures (Klibi \& Martel, 2013) like Conditional Value-At-Risk and Value-At-Risk of the expected cost of the SC and cost variance. Models with robust measures like $\mathrm{p}$ robustness, min-max cost and minimizing relative regret are limited. Our model minimizes expected cost and time such that the expected cost of reconfiguration is restricted in each disruption scenario by a user- defined threshold through $\mathrm{p}$ robustness measure in the constraint (Peng et al., 2011).Thereby a robust SCND is generated before realization of any scenario that will perform efficiently under any disruption scenario after reconfiguring with optimal reactive strategy.

Finally, a number of solution approaches to the SCNR models have been proposed: commercial mathematical programming software (Melo et al., 2006), branch and bound (Huang \& Goetschalckx, 2014), Benders decomposition (Kristianto et al., 2014), Lagrangian relaxation [22,29] and heuristics (Melo et al., 2012). Since commercial solvers are highly suitable for small and medium problem instances and heuristics and meta-heuristics are preferred for large scale problems to reduce computational time (Bashiri et al., 2012), our model uses the augmented $\varepsilon$-constraint method (Mavrotas \& Florios, 2013) coded in GAMS 23.5 as a solution approach. The rest of the paper is organised as follows. In section 2, a multi-objective SCNR model 
is formulated. An illustrative example is presented in section 3 . The results obtained using the augmented $\varepsilon$-constraint method is detailed in section 4 . In section 5 , conclusions and future research are discussed.

\section{Problem description and model formulation}

We model a three echelon supply chain network consisting of plants, transhipments and customers where a single product is delivered to various demand points over a finite planning horizon and the network is subjected to facility disruptions (Peng et al, 2011). Simultaneous facility disruptions are rare enough to be negligible (Schmitt, 2011). Disruption of single facility at a time is considered in our work. It is assumed that disruption is complete and once a facility is disrupted it doesn't recover. The planning horizon is assumed to cover a set of period $t \in(0,1$, $2 \ldots \mathrm{T})$. Along this horizon disruption may occur and reconfiguration decisions are made only in the planning periods $\operatorname{td}^{\mathrm{t}} \in \mathrm{T}$. This disruption reconfiguration planning periods are assumed to be three periods following the works of Hendricks \& Singhal, (2005), Losada et al., (2010, 2012), Sahebjamnia et al., (2015) and Torabi et al., (2015). The first period within reconfiguration planning horizon when disruption occurs is termed as 'onset period' and the remaining two periods are termed as 'recovery periods' as shown in figure 1.As our problem is long term strategic in nature where each period may represent one or more years, inventory decisions do not provide representative information and are not considered in the model i.e. material inflow at a facility is equal to its outflow (Pimentel et al., 2010).

The facility location decisions are made at the beginning of planning horizon $\left(\mathrm{t}_{0}\right)$ and remain unchanged. When the network is disrupted at period $\mathrm{td}^{1}$, optimal reactive reconfiguration is arrived. Within reconfiguration planning horizon, unmet demands are satisfied either by shifting sourcing/ production to different undisrupted facilities, or by expanding capacity at selected facilities or by outsourcing to external facility. Outsourced products are directly delivered to customers. The capacity of external facility is assumed to be infinite (Cortinhal et al., 2015; Thanh et al., 2011, 2010,2008; Hinojosa et al., 2008).Capacity expansion at undisrupted facilities is not permitted during disruption onset period $\mathrm{td}^{1}$ as it is not practically feasible to implement structural changes in short term immediately after the disruption (Melo etal., 2006 and Ivanov et al., 2016).

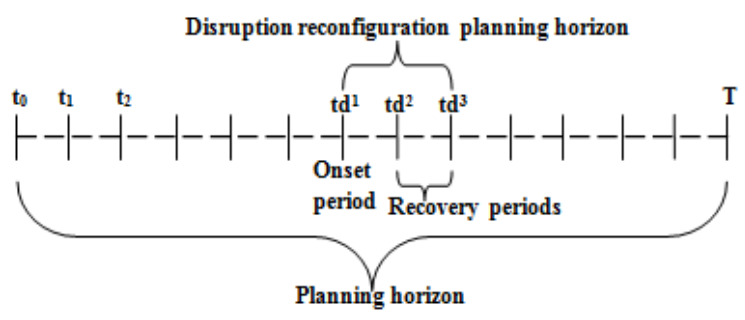

Figure 1: SCN reconfiguration and planning horizon

The specifications of the model are given below.

\section{Indices}

p Plants $(\mathrm{p}=1,2, \ldots . \mathrm{P})$

i Transhipment $(\mathrm{i}=1,2, \ldots \mathrm{I})$

1 Customers $(1=1,2, \ldots . \mathrm{L})$

t Planning horizon $(\mathrm{t}=1,2, \ldots \mathrm{T})$

s $\quad$ Scenarios $(\mathrm{s}=1,2, \ldots \mathrm{S})$

$\boldsymbol{p} \boldsymbol{d}_{\boldsymbol{s}}^{\boldsymbol{p}}$ Set of disrupted plants ' $\mathrm{p}$ ' in scenario's'

$\boldsymbol{i d} \boldsymbol{d}_{\boldsymbol{s}}^{i}$ Set of disrupted transhipments ' $\mathrm{i}$ ' in scenario's'

$\boldsymbol{t} \boldsymbol{d}_{\boldsymbol{s}}^{\boldsymbol{t}}$ Set of disruption reconfiguration planning periods ' $t$ ' in scenario' $s$ '

$\boldsymbol{t} \boldsymbol{r}_{\boldsymbol{s}}^{t}$ Set of recovery periods 't' in scenario's' $\left(t r_{s}^{t} \subset t d_{s}^{t}\right)$

\section{Parameters}

$\boldsymbol{D}_{\boldsymbol{t l}}$ Demand at customer 1 in period $\mathrm{t}$

$\overline{\boldsymbol{K}}_{\boldsymbol{p}}$ Initial capacity of plant $\mathrm{p}$

$\overline{\boldsymbol{K}}_{\boldsymbol{i}}$ Initial capacity of transhipment i

$\overline{\overline{\boldsymbol{K}}}_{\boldsymbol{p}}$ Maximum allowed capacity at plant $\mathrm{p}$

$\overline{\overline{\boldsymbol{K}}}_{\boldsymbol{i}}$ Maximum allowed capacity at transhipment i

$\boldsymbol{q}_{\boldsymbol{s}}$ Probability of scenario s

$\boldsymbol{f}_{\boldsymbol{p}}$ Fixed cost of plant $\mathrm{p}$

$\boldsymbol{f}_{\boldsymbol{i}}$ Fixed cost of transhipment $\mathrm{i}$

$\boldsymbol{C} \boldsymbol{t}_{\boldsymbol{p i}}$ Unit transportation cost from plant $\mathrm{p}$ to transhipment i

$\boldsymbol{C} \boldsymbol{t}_{\boldsymbol{i l}}$ Unit transportation cost from transhipment i to customer 1

$\boldsymbol{C} \boldsymbol{p} \boldsymbol{t}_{\boldsymbol{l}}$ Unit transportation cost from external facility to customer 1

$\boldsymbol{C} \boldsymbol{n}_{\boldsymbol{t} \boldsymbol{p}}$ Unit new capacity addition cost at plant $\mathrm{p}$ in period $\mathrm{t}$

$\boldsymbol{C} \boldsymbol{n}_{\boldsymbol{t} i}$ Unit new capacity addition cost at transhipment $\mathrm{i}$ in period $\mathrm{t}$ 
$\boldsymbol{T} \boldsymbol{t}_{\boldsymbol{p} \boldsymbol{i}}$ Transportation time from plant $\mathrm{p}$ to transhipment $\mathrm{i}$

$\boldsymbol{T} \boldsymbol{t}_{\boldsymbol{i l}}$ Transportation time from transhipment i to customer 1

$\boldsymbol{T} \boldsymbol{t}_{\boldsymbol{l}}$ Transportation time from external facility to customer 1

$\boldsymbol{f} \boldsymbol{s}_{\text {stp }}$ Failure state of plant $\mathrm{p}$ in period $\mathrm{t}$ and in scenario $s\left\{\begin{array}{l}1, \text { if plant } \mathrm{p} \text { disrupted } \\ 0, \quad \text { Otherwise }\end{array}\right.$

$\boldsymbol{f} \boldsymbol{s}_{\boldsymbol{s} t \boldsymbol{i}}$ Failure state of transhipment $\mathrm{i}$ in period $\mathrm{t}$ and in scenario s $\{1$, if transshipment i disrupted $\boldsymbol{\text { maxt }}_{\boldsymbol{t p i}}, \boldsymbol{m i n t}_{\boldsymbol{t} \boldsymbol{p i}} \mathbf{M}^{\text {Maximum and minimum limit of }}$ quantity of shipped from $\mathrm{p}$ to i in period tmaxt $\boldsymbol{t}_{t i}$ quantity of shipped from i to $\mathrm{l}$ in period $\mathrm{t}$ $\boldsymbol{m a x n}_{t \boldsymbol{p}}, \boldsymbol{m i n n}_{\boldsymbol{t} \boldsymbol{p}}$ Maximum and minimum limit of adding capacity at plant $\mathrm{p}$ in period $\mathrm{t}$

$\boldsymbol{m a x n}_{\boldsymbol{t i}}, \boldsymbol{m i n n}_{\boldsymbol{t} i}$ Maximum and minimum adding capacity at transhipment $i$ in period $t$ $\boldsymbol{m} \boldsymbol{e}_{\boldsymbol{p}}, \boldsymbol{m} \boldsymbol{e}_{\boldsymbol{i}}$ Minimum capacity utilisation for a facility $\mathrm{p} / \mathrm{i}$ if opened

\section{Decision Variables}

$\boldsymbol{Q t}_{\text {stpi }}$ Quantity transported from plant p to transhipment $\mathrm{i}$ in period $\mathrm{t}$ and in scenario $\mathrm{s}$

$\boldsymbol{Q t}_{\text {stil }}$ Quantity transported from transhipment i to customer $\mathrm{l}$ in period $\mathrm{t}$ and in scenario $\mathrm{s}$

$\boldsymbol{Q} \boldsymbol{n}_{\boldsymbol{s t} \boldsymbol{p}} \mathrm{Quantity}$ of capacity added at plant $\mathrm{p}$ in period $\mathrm{t}$ under scenario $\mathrm{s}$

$\boldsymbol{Q} \boldsymbol{n}_{\boldsymbol{s t i}}$ Quantity of capacity added at transhipment i in period $t$ under scenario $s$

$\boldsymbol{Q p}_{\boldsymbol{s t l}} \mathrm{Quantity}$ supplied by external facility for customer 1 in period $t$ in scenario $\mathrm{s}$

$\boldsymbol{x}_{\boldsymbol{p}}\left\{\begin{array}{lr}1, & \text { if plant p opens } \\ 0, & \text { Otherwise }\end{array}\right.$

$\boldsymbol{x}_{\boldsymbol{i}}\left\{\begin{array}{lr}1, & \text { if transhipment i opens } \\ 0, & \text { Otherwise }\end{array}\right.$

$\boldsymbol{q} \boldsymbol{t}_{\text {stpi }}\left\{\begin{array}{c}1, \text { if plant } \mathrm{p} \text { transports to } \\ \text { transshipment i in period } \mathrm{t} \text { and scenario } \mathrm{s} \\ 0, \quad \text { Otherwise }\end{array}\right.$

(1, if transhipment i transports to

$\boldsymbol{q} \boldsymbol{t}_{\text {stil }}\left\{\begin{array}{c}\text { customer } \mathrm{l} \text { in period } \mathrm{t} \text { and scenario s } \\ 0, \quad \text { Otherwise }\end{array}\right.$

$\boldsymbol{q n}_{\text {stp }}\left\{\begin{array}{c}1, \text { if new capacity is built at plant } \mathrm{p} \\ \text { in period t and scenario s } \\ 0, \quad \text { Otherwise }\end{array}\right.$

$\boldsymbol{q} \boldsymbol{n}_{\boldsymbol{s t i}}\left\{\begin{array}{c}1, \text { if new capacity is built at transshipment i } \\ \text { in period t and scenario s } \\ 0, \quad \text { Otherwise }\end{array}\right.$

$\boldsymbol{q p}_{\text {stl }}\left\{\begin{array}{c}1, \text { if demand of customer l is met by external } \\ \text { facility in period t and scenario s } \\ 0, \quad \text { Otherwise }\end{array}\right.$
Mathematical model

$$
\begin{aligned}
& \text { Minimise } \\
& \sum_{p \in P} f_{p} x_{p}+\sum_{i \in I} f_{i} x_{i} \\
& +\sum_{s \in S} q_{s}\left(\sum_{t \in T} \sum_{p \in P} \sum_{i \in I} Q t_{s t p i} C t_{p i}\right. \\
& +\sum_{t \in T} \sum_{i \in I} \sum_{l \in L} Q t_{s t i l} C t_{i l} \\
& +\sum_{t \in T} \sum_{p \in P} Q n_{s t p} C n_{t p}
\end{aligned}
$$

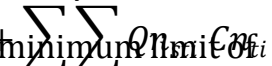

$$
\begin{aligned}
& \left.+\sum_{t \in T}^{t \in T} \sum_{l \in L}^{i \in I} Q p_{s t l} C p_{t l}\right)
\end{aligned}
$$

\section{Minimise}

$$
\begin{aligned}
\sum_{s \in S} q_{s}\left(\sum_{t \in T} \sum_{p \in P} \sum_{i \in I} T t_{p i} q t_{s t p i}\right. \\
+\sum_{t \in T} \sum_{i \in I} \sum_{l \in L} T t_{i l} q t_{s t i l} \\
+\sum_{t \in T} \sum_{l \in L}^{\left.T t_{l} q p_{s t l}\right)(\mathbf{2})}
\end{aligned}
$$

Subjected to constraints

$$
\begin{aligned}
& \sum_{p \in P} f_{p} x_{p}+\sum_{i \in I} f_{i} x_{i}+\sum_{t \in T} \sum_{p \in P} \sum_{i \in I} Q t_{s t p i} C t_{p i} \\
& +\sum_{t \in T} \sum_{i \in I} \sum_{l \in L} Q t_{s t i l} C t_{i l} \\
& +\sum_{t \in T} \sum_{p \in P} Q n_{s t p} \\
& +\sum_{t \in T} \sum_{i \in I} Q n_{s t i} C n_{t i} \\
& +\sum_{t \in T} \sum_{l \in L} Q p_{s t l} C p_{t l} \\
& \leq(1+p) C_{s}^{*} \forall \boldsymbol{s}(\mathbf{3}) \\
& \sum_{i \in I} Q t_{s t i l}+Q p_{s t l}=D_{t l} \forall \boldsymbol{s} \in \boldsymbol{S}, \boldsymbol{t} \in \boldsymbol{T}, \boldsymbol{l} \in \boldsymbol{L}(\mathbf{4}) \\
& \sum_{i \in I} Q t_{s t p i} \leq\left(\bar{K}_{p} x_{p}+\sum_{\tau=1}^{t} Q n_{s \tau p}\right)\left(1-f s_{s t p}\right) \\
& \forall \boldsymbol{s} \in \boldsymbol{S}, \boldsymbol{t} \in \boldsymbol{T} \boldsymbol{p} \in \boldsymbol{P} \\
& \sum_{i \in I} Q t_{s t p i} \geq M e_{p}\left(\bar{K}_{p} x_{p}+\sum_{\tau=1}^{t} Q n_{s \tau p}\right)\left(1-f s_{s t p}\right) \\
& \forall s \in S, t \in T p \in P \\
& \sum_{l \in L} Q t_{s t i l} \leq\left(\bar{K}_{i} x_{i}+\sum_{\tau=1}^{t} Q n_{s \tau i}\right)\left(1-f s_{s t i}\right) \\
& \forall \boldsymbol{s} \in \boldsymbol{S}, \boldsymbol{t} \in \boldsymbol{T} \boldsymbol{i} \in \boldsymbol{I}
\end{aligned}
$$




$$
\begin{aligned}
& \sum_{l \in L} Q t_{s t i l} \geq M e_{i}\left(\bar{K}_{i} x_{i}+\sum_{\tau=1}^{t} Q n_{s t i}\right)\left(1-f s_{s t i}\right) \\
& \forall s \in S, t \in T i \in I \\
& \sum_{l \in L} Q t_{s t i l}=\sum_{p \in P} Q t_{s t p i} \forall \boldsymbol{s} \in \boldsymbol{S}, \boldsymbol{t} \in \boldsymbol{T}, \boldsymbol{i} \in \boldsymbol{I} \\
& Q n_{s t p}=0 \quad \forall \boldsymbol{s} \in \boldsymbol{S}, \boldsymbol{t} \in \boldsymbol{T}, \boldsymbol{p} \in \boldsymbol{p} \boldsymbol{d}_{\boldsymbol{s}}^{\boldsymbol{p}} \\
& Q n_{s t i}=0 \quad \forall s \in S, t \in T, i \in i d_{s}^{i} \\
& Q n_{s t p}=0 \quad \forall \boldsymbol{s} \in \boldsymbol{S}, \boldsymbol{t} \in \boldsymbol{T} \backslash \boldsymbol{t} \boldsymbol{r}_{\boldsymbol{s}}^{\boldsymbol{t}}, \boldsymbol{p} \in \boldsymbol{P} \\
& Q n_{s t i}=0 \forall \boldsymbol{s} \in \boldsymbol{S}, \boldsymbol{t} \in \boldsymbol{T} \backslash \boldsymbol{t} \boldsymbol{r}_{s}^{t}, \boldsymbol{i} \in \boldsymbol{I} \\
& Q p_{s t l}=0 \quad \forall \boldsymbol{s} \in \boldsymbol{S}, \boldsymbol{t} \in \boldsymbol{T} \backslash \boldsymbol{t} \boldsymbol{d}_{\boldsymbol{s}}^{\boldsymbol{t}}, \boldsymbol{l} \in \boldsymbol{L} \\
& Q t_{\text {stpi }} \leq \operatorname{maxt}_{t p i} q t_{\text {stpi }} \\
& \forall \boldsymbol{s} \in \boldsymbol{S}, \boldsymbol{t} \in \boldsymbol{T}, \boldsymbol{p} \in \boldsymbol{P}, \boldsymbol{i} \in \boldsymbol{I} \\
& Q t_{\text {stpi }} \geq \operatorname{mint}_{\text {tpi }} q t_{\text {stpi }} \\
& \forall \boldsymbol{s} \in \boldsymbol{S}, \boldsymbol{t} \in \boldsymbol{T}, \boldsymbol{p} \in \boldsymbol{P}, \boldsymbol{i} \in \boldsymbol{I} \\
& Q t_{\text {stil }} \leq \operatorname{maxt}_{\text {til }} q t_{\text {stil }} \\
& \forall s \in S, t \in T, i \in I, l \in L \\
& Q t_{\text {stil }} \geq \operatorname{mint}_{\text {til }} q t_{\text {stil }} \\
& \forall s \in S, t \in T, i \in I, l \in L \\
& Q n_{s t p} \leq \operatorname{maxn}_{t p} q n_{s t p} \\
& \forall \boldsymbol{s} \in \boldsymbol{S}, \boldsymbol{t} \in \boldsymbol{T}, \boldsymbol{p} \in \boldsymbol{P} \\
& Q n_{s t p} \geq \operatorname{minn}_{t p} q n_{s t p} \\
& \forall s \in S, t \in T, p \in P \\
& Q n_{s t i} \leq \operatorname{maxn}_{t i} q n_{s t i} \\
& \forall s \in S, t \in T, i \in I \\
& Q n_{s t i} \geq \operatorname{minn}_{t i} q n_{s t i} \\
& \forall s \in S, t \in T, i \in I \\
& Q p_{s t l} \leq D_{t l} q n_{s t l} \\
& \forall s \in S, t \in T, l \in L \\
& q t_{s t p i} \leq x_{p} \forall \boldsymbol{s} \in \boldsymbol{S}, \boldsymbol{t} \in \boldsymbol{T}, \boldsymbol{p} \in \boldsymbol{P}, \boldsymbol{i} \in \boldsymbol{I} \text { (24) } \\
& q t_{s t i l} \leq x_{i} \forall s \in S, t \in T, i \in I, l \in L \text { (25) } \\
& q n_{s t p} \leq x_{p} \forall \boldsymbol{s} \in \boldsymbol{S}, \boldsymbol{t} \in \boldsymbol{T}, \boldsymbol{p} \in \boldsymbol{P} \text { (26) } \\
& q n_{s t i} \leq x_{i} \forall \boldsymbol{s} \in \boldsymbol{S}, \boldsymbol{t} \in \boldsymbol{T}, \boldsymbol{i} \in \boldsymbol{I} \\
& Q t_{\text {stpi }}, Q t_{\text {stil }}, Q n_{s t p}, Q n_{s t i} \geq 0 \\
& \forall s \in S, t \in T, p \in P, i \in I \\
& q t_{s t p i}, q t_{s t i l}, q n_{s t p}, q n_{s t i} \in 1,0 \\
& \forall s \in S, t \in T, p \in P, i \in I
\end{aligned}
$$

The objective function (1) minimizes the expected SCNR cost which consists of fixed costs of plants and transhipments, transportation costs from plants to transhipments and from transhipments to customers, capacity expansion cost and cost of outsourcing from external facility. Among the cost components, only the fixed cost of plants and transhipments are scenario and time independent. Objective function (2) minimizes the expected delivery time consisting of transportation time from plants to transhipments, from transhipments to customers and from external facility to customers. Transportation times between echelons are independent of quantity transported. Constraint (3) enforce the p-robustness criterion, requiring that the relative regret for scenario's' may not be more than $\mathrm{p} \%$ of deterministic optimal scenario cost. Let $\mathrm{O}(\mathrm{s})$ be the deterministic cost minimisation problem for each scenario's' given in appendix $\mathrm{A} 1$ and $C_{s}^{*}$ be the corresponding objective value for the problem. Constraint (4) states that customer demand can be met using products from the plants and outsourcing from external facility. Constraints (5 and 7) ensure that the total out-flow from a facility (plant and transhipment) does not exceed its capacity when opened and undisrupted in that scenario, and prevent any flow when it is closed or disrupted. Further, the second term on right side indicates the added new capacity after expansion. The set ' $\tau$ ' is an alias of set ' $t$ '. Constraints (6 and 8) ensure that a facility (plant and transhipment)is opened only if a least specified capacity is used. Constraint (9) ensures the material flow constraint for transhipment nodes. Constraint set (10 and 11) restricts adding new capacity at disrupted facility (plant and transhipment)and Constraint set (12 and 13) restricts adding new capacity during the planning periods other than disruption recovery periods. Constraint 14 ensures that outsourcing is not opted outside the reconfiguration planning horizon. Constraints (15-18 and 19-22) restrict the transportation arc capacity and capacity addition per period at facilities. Constraint 23 restricts maximum outsourced quantity to be less than corresponding customer's demand. Constraint set (24-27) ensures that product flow and capacity expansion occur only in opened facilities. Constraints (28) and (29) are standard integrity and non negativity constraints.

\section{Numerical example}

We now demonstrate the proposed model, using data drawn from the literature where possible and available. A 3 echelon SC with 5 plants, 7 transshipments and 20 customers is considered. The planning horizon is assumed to have seven periods $\left(t_{0} t_{0} t_{6}\right)$. The disruption scenarios are assumed to occur at period $t_{2}$ and hence reconfiguration planning periods include periodst $t_{2}$ to $t_{4}$. A set of twelve scenarios with single facility disruption are generated. Scenarios s1-s5 considers plant disruption and scenarios s6-s12 consider transshipment disruptions. 
The probabilities of scenarios are uniformly generated between $(0,1)$.

Following Peng et al., (2011), each customer demand is uniformly drawn from $(50,110)$. We calculate the total customer demands in the first period. This sum demand is then divided by $|\mathrm{i}|$ to determine average required supply from transshipments. The initial capacity of transshipment node is drawn uniformly from $(2.5,3)$ of average required supply. Same procedure is followed for calculating initial capacity of plants. Following Thanh et al., 2008, the fixed cost for a facility is uniformly drawn from $(60,65)$ of initial capacity. The capacity expansion cost per unit (Melo et al., 2006) takes a value uniformly between $(25,50)$. The distance between facilities is drawn uniformly from $(1,250)$. The transportation is assumed by road and the average transportation cost is assumed as INR Rs. 2/unit distance/unit quantity. The delivery time between source and destination nodes is assumed to be proportional to the distance between them.

Maximum allowable facility capacity after expansion is restricted to 1.5 times the initial capacity. Each facility per period can add no more than and no less than $1 / 4$ and $1 / 12$ of its initial capacity (Thanh et al., 2008). The minimum capacity utilization for a facility to be opened is assumed as 20\% (Badri et al., 2013). The outsourcing cost and time are given a large value of INR 2500 and 500 days respectively (Peng et al., 2011).The value of 'p' in robustness constraint is user-defined and is set as 0.22 .

\section{Results and discussions}

The proposed model is solved using augmented $\varepsilon$ constraint method (Mavrotas, 2006) and is coded using GAMS 23.5.The Pareto front obtained is shown in figure 2.

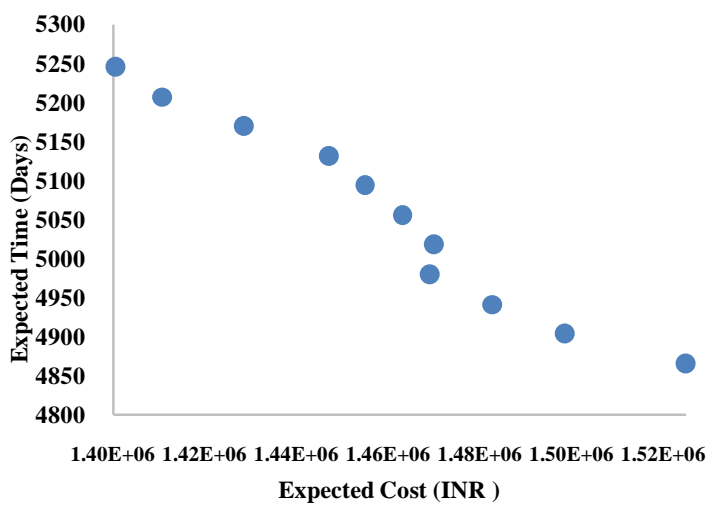

Fig. 2: The Pareto Front for the problem

The nominal scenario cost and time are INR 1458202 and 5159 days respectively. The results are further reported in terms of choice and capacity utilization of opened facilities after disruption with respect to generated Pareto solutions.

The Pareto solutions with the details of opened facilities are shown in table 1. In the obtained Pareto solutions, all plants 'p1-p4'are opened except in the minimum cost solution and in a solution closer to ideal solution (1405793, 5207.09) identified following the work of Prasanna Venkatesan and Kumanan (2012). Similarly in all the solutions the transshipments 'i1'- ‘i7' are opened.

Table 1: Pareto solutions with choice of facilities

\begin{tabular}{|c|c|c|c|}
\hline \multicolumn{2}{|c|}{ Expected } & \multicolumn{2}{c|}{ Facilities opened } \\
\hline Cost (INR) & Time (Days) & Plants & Transshipment \\
\hline 1395584 & 5244.89 & $2,3,4$ & $1,2,3,4,5,6,7$ \\
\hline 1519998 & 4865.38 & $1,2,3,4$ & $1,2,3,4,5,6,7$ \\
\hline 1405793 & 5207.09 & $2,3,4$ & $1,2,3,4,5,6,7$ \\
\hline 1423687 & 5169 & $1,2,3,4$ & $1,2,3,4,5,6,7$ \\
\hline 1442111 & 5131.08 & $1,2,3,4$ & $1,2,3,4,5,6,7$ \\
\hline 1449894 & 5093 & $1,2,3,4$ & $1,2,3,4,5,6,7$ \\
\hline 1458064 & 5055.29 & $1,2,3,4$ & $1,2,3,4,5,6,7$ \\
\hline 1465111 & 5017.38 & $1,2,3,4$ & $1,2,3,4,5,6,7$ \\
\hline 1464141 & 4979.4 & $1,2,3,4$ & $1,2,3,4,5,6,7$ \\
\hline 1477787 & 4941.45 & $1,2,3,4$ & $1,2,3,4,5,6,7$ \\
\hline 1493654 & 4903.5 & $1,2,3,4$ & $1,2,3,4,5,6,7$ \\
\hline
\end{tabular}

The percentage of customer demand allocated to opened facilities and their capacity utilization during period ' $t_{0}$ ' in nominal scenario ' $\mathrm{s} 0$ ' are show in table 2 for extreme solutions and solution closer to ideal point respectively. It is observed from table 2 that nearly $50 \%$ of customer's total demand is satisfied by plant ' $\mathrm{p} 3$ ' at nominal scenario in all the three solutions. This is due to the fact that ' $\mathrm{p} 3$ ' is closer to all transshipment nodes and hence the variable transportation cost and delivery time are minimized. 
Table 2: Percentage customer demand allocated to opened facilities and their capacity utilization during period 'to' in nominal scenario 's0' for MC, MT and IP solutions

\begin{tabular}{|c|c|c|c|c|c|c|c|c|c|c|c|c|}
\hline $\begin{array}{c}\text { Pareto } \\
\text { solutions }\end{array}$ & \multicolumn{4}{|c|}{ MC } & \multicolumn{4}{|c|}{ MT } & \multicolumn{4}{|c|}{ IP } \\
\hline \multirow[t]{2}{*}{ Periods } & \multicolumn{2}{|c|}{$\begin{array}{l}\text { Location with percentage of } \\
\text { customer demand } \\
\text { allocation of opened }\end{array}$} & \multicolumn{2}{|c|}{$\begin{array}{c}\text { Capacity Utilization of } \\
\text { opened }\end{array}$} & \multicolumn{2}{|c|}{$\begin{array}{c}\begin{array}{c}\text { Location with percentage of } \\
\text { customer demand allocation } \\
\text { of opened }\end{array} \\
\end{array}$} & \multicolumn{2}{|c|}{$\begin{array}{c}\text { Capacity Utilization of } \\
\text { opened }\end{array}$} & \multicolumn{2}{|c|}{$\begin{array}{c}\text { Location with percentage of } \\
\text { customer demand allocation of } \\
\text { opened }\end{array}$} & \multicolumn{2}{|c|}{$\begin{array}{c}\text { Capacity Utilization of } \\
\text { opened }\end{array}$} \\
\hline & Plants & Transshipments & Plants & Transshipments & Plants & Transshipments & Plants & Transshipments & Plants & Transshipments & Plants & Transshipments \\
\hline \multirow{7}{*}{$\mathrm{t}_{0}$} & \multirow{2}{*}{ p2-19.3 } & i1-9.1 & \multirow{2}{*}{ p2-0.366 } & i1-0.242 & \multirow{2}{*}{ p1-17.9 } & i1-17.9 & \multirow{2}{*}{ p1-0.296 } & i1-0.475 & \multirow{2}{*}{ p2-19.3 } & i1-9.1 & \multirow{2}{*}{ p2-0.366 } & i1-0.242 \\
\hline & & i2- 12.8 & & $\mathrm{i} 2-0.333$ & & i2-12.8 & & i2- 0.333 & & i2- 12.8 & & i2 $2-0.333$ \\
\hline & \multirow{2}{*}{ p3-55.7 } & i3-14.3 & \multirow{2}{*}{ p3-0.974 } & i3-0.38 & \multirow{2}{*}{ p2-10.6 } & i3--10 & \multirow{2}{*}{ p2-0.201 } & i3-0.266 & \multirow{2}{*}{ p3-55.7 } & i3-14.3 & \multirow{2}{*}{ p3-0.974 } & i3-0.38 \\
\hline & & i4-19.3 & & i4-0.507 & & i4-10.6 & & i4-0.279 & & i4-19.3 & & i4-0.507 \\
\hline & \multirow{3}{*}{$\mathrm{p} 4-25$} & i5-10.5 & \multirow{3}{*}{ p4-0.428 } & i5-0.257 & \multirow{3}{*}{$\begin{array}{l}\text { p3-42.3 } \\
\text { p4-29.2 }\end{array}$} & i5-10.5 & \multirow{3}{*}{$\begin{array}{c}\mathrm{p} 3-0.739 \\
\mathrm{p} 4-0.52\end{array}$} & i5-0.257 & & i5-10.5 & & i5-0.257 \\
\hline & & i6-21.8 & & i6-0.523 & & i6-21.8 & & i6- 0.523 & $\mathrm{p} 4-25$ & i6-21.8 & p4-0.428 & i6-0.523 \\
\hline & & i7-12.2 & & i7-0.33 & & i7-17.5 & & i7-0.476 & & i7-12.2 & & i7-0.33 \\
\hline & 0208 & i1-10.1 & 020.377 & i1- 0.253 & 105 & i1-19.5 & 010305 & i1- 0.488 & 20 & i1-16.1 & 200201 & i1-0.401 \\
\hline & $\mathrm{p} 2-20.0$ & i2 -9.4 & $\mathrm{p} z-0.0 / 2$ & $\mathrm{i} 2-0.232$ & $p 1-19 . J$ & i2 -9.4 & p1-0.505 & $\mathrm{i} 2-0.232$ & $\mathrm{p} 2-11.2$ & i2 -9.4 & $\mathrm{p} 2-0.201$ & i2- -0.232 \\
\hline & & i3-12 & & i3-0.302 & & i3-8.6 & & i3-0.214 & $n 3-50$ & i3-12 & n3-0.97 & i3-0.302 \\
\hline $\mathrm{t}_{2}$ & p3-59.2 & i4-20.8 & p3-0.9/5 & i4-0.516 & $\mathrm{p} 2-11.4$ & i4-11.4 & $\mathrm{p} 2-0.205$ & i4- 0.284 & p3-5y & i4-0 & ps -0.91 & i4-0 \\
\hline & & i5-9.7 & & i5-0.223 & & i5-9.7 & & i5-0.223 & & i5-14.7 & & i5-0.339 \\
\hline & p4-19.9 & i6--27.4 & p4-0.322 & i6-0.62 & p3-45.6 & i6-27.4 & p3-0.751 & i6-0.62 & p4-29.8 & i6-27.4 & p4-0.481 & i6-0.62 \\
\hline & & i7-10.5 & & i7-.269 & & i7-13.9 & & i7-0.356 & & i7-20.3 & & i7-0.521 \\
\hline & $n^{2}-205$ & i1-8.8 & $p^{2} 0358$ & i1-0.214 & n1-212 & i1-21.2 & n10320 & i1-0.515 & $p^{2}-115$ & i1-14.6 & 201 201 & i1-0.356 \\
\hline & $\mathrm{p} 2-20.3$ & i2-10.1 & $p 2-0.530$ & i2- -0.241 & $p_{1-21.2}$ & i2-10.1 & $p 1-0.522$ & i2- 0.241 & $02-11 . J$ & i2-10.1 & $p 2-0.201$ & i2- -0.241 \\
\hline & & i3-17.4 & & i3- -0.424 & & i3-10.9 & & i3-0.265 & & i3-17.4 & & i3-0.424 \\
\hline$t_{3}$ & p3- 59.8 & i4-20.5 & p3-0.958 & i4-0.496 & p2- 11.5 & i4-11.5 & $\mathrm{p} 2-0.201$ & i4-0.279 & p3-57.8 & i4-0 & p3-0.926 & i4-0 \\
\hline & & i5-9.4 & & i5-0.211 & & i5-9.4 & & i5-0.211 & & i5-13.1 & & i5-0.294 \\
\hline & p4-19.7 & i6-24.2 & p4-0.309 & i6-0.533 & p3-44.5 & i6-24.2 & p3-0./13 & i6-0.533 & p4-30.7 & i6-24.2 & $\mathrm{p} 4-482$ & i6-0.533 \\
\hline & & i7-9.6 & & i7-.238 & & i7-14 & & i7-0.349 & & i7-20.5 & & i7-0.512 \\
\hline & & i1-7.9 & & i1-0.201 & & i1-23.3 & & i1-0.589 & & i1-18 & & i1- 0.455 \\
\hline & $\mathrm{p} 2-24.1$ & i2- 8.1 & $\mathrm{p} 2-0.437$ & i2-0.2 & pl-23.3 & i2-8.1 & p1-0.36/ & i2-0.2 & $\mathrm{p} 2-11.1$ & i2-8.1 & $\mathrm{p} 2-0.201$ & i2-0.2 \\
\hline & & i3-14 & & i3-0.353 & & i3-10.4 & & i3-0.263 & & i3-15.7 & & i3-0.397 \\
\hline $\mathrm{t}_{4}$ & p3- 58.9 & i4-24.1 & p3-0.98 & i4-0.605 & $\mathrm{p} 2-11.1$ & i4-11.1 & p2-0.201 & i4- 0.279 & p3-63.6 & i4-0 & p3-1.059 & i4-0 \\
\hline & & i5-12.2 & & i5-0.284 & & i5-12.2 & & i5-0.284 & & i5-16.3 & & i5-0.379 \\
\hline & p4- 17.2 & i6-24.8 & p4-0.281 & i6-0.568 & p3-47.4 & i6-24.8 & $\begin{array}{l}p-0.189 \\
0.0338\end{array}$ & i6-0.568 & $\mathrm{p} 4-25.5$ & i6-24.8 & p4-0.416 & i6-0.568 \\
\hline & & i7-9.1 & & i7-0.236 & & i7-12.6 & p4-0.538 & i7-0.327 & & i7-17.4 & & i7-0.45 \\
\hline
\end{tabular}


During the disruption onset period ' $\mathrm{t}_{2}$ ' where capacity expansion is not feasible, the unmet demands are fulfilled mainly by shifting them to least utilized facilities considering the transportation cost and delivery time. For example, in the minimum time solution, when plant ' $\mathrm{p} 4$ ' gets disrupted the underutilized facilities ' $\mathrm{p} 1$ ' and ' $\mathrm{p} 2$ ' serves the disrupted facility's demand ( $23.4 \%$ of total demand). It is found that the undisrupted facility ' $\mathrm{p} 1$ ' serves $20 \%$ of the demand while ' $\mathrm{p} 2$ ' serves the remaining 3.4\% considering the transportation cost and delivery time. Also it is observed that during the recovery periods ' $t_{3}$ ' and ' $t_{4}$ ', capacity expansion occurs at facility ' $\mathrm{p} 3$ ' which is close to all transshipment nodes. More capacity expansion occurred in minimum cost solution as more number of facilities are opened in minimum time solution than minimum cost solution. Similarly the capacity utilization of facilities in minimum cost solutions are higher compared to that of minimum time solution.

\section{Conclusion}

We have developed a multi-objective SCN reconfiguration in a dynamic planning horizon under facility disruptions. The reconfiguration decisions considered are shifting customer demands to undisrupted facilities, outsourcing to external facility and capacity expansion at undisrupted facilities. The augmented $\varepsilon$-constraint method is proposed as a solution approach to obtain a set of Pareto optimal solutions of minimised expected network cost and delivery time. Numerical illustrations with findings are presented. One possible extension of our model is to study the reconfiguration decisions of more than one facility disruptions. Another direction for improvement could be consideration of partial facility disruptions and capacity recovery of disrupted facilities over a time period.

\section{Acknowledgement}

The authors acknowledge DST-FIST for sponsoring the purchase of GAMS software vide sanction order No. SR/FST/ETI-259/2009.

\section{Appendix}

A1:The optimal scenario costs $C_{s}^{*}$ are calculated by solving the model $\mathrm{O}(\mathrm{s})$ for each of the scenario's'.
$\mathbf{O}(\mathbf{s})$

$$
\begin{aligned}
& \sum_{p \in P}^{C_{s}^{*}}=\text { Minimise } \\
& f_{p} x_{p}+\sum_{i \in I} f_{i} x_{i} \\
& +\sum_{t \in T} \sum_{p \in P} \sum_{i \in I} Q t_{s t p i} C t_{p i}+\sum_{t \in T} \sum_{i \in I} \sum_{l \in L} Q t_{s t i l} C t_{i l} \\
& +\sum_{t \in T} \sum_{p \in P} Q n_{s t p} C n_{t p}+\sum_{t \in T} \sum_{i \in I} Q n_{s t i} C n_{t i} \\
& +\sum_{t \in T} \sum_{l \in L} Q p_{s t l} C p_{t l}
\end{aligned}
$$

Subjected to constraints

$$
\begin{aligned}
& \sum_{i \in I} Q t_{s t i l}+Q p_{s t l}=D_{t l} \forall \boldsymbol{t} \in \boldsymbol{T}, \boldsymbol{l} \in \boldsymbol{L}(\mathbf{3 1}) \\
& \sum_{i \in I} Q t_{s t p i} \leq\left(\bar{K}_{p} x_{p}+\sum_{\tau=1}^{t} Q n_{s \tau p}\right)\left(1-f s_{s t p}\right) \\
& \forall \boldsymbol{t} \in \boldsymbol{T} \boldsymbol{p} \in \boldsymbol{P} \\
& \sum_{i \in I} Q t_{s t p i} \geq M e_{p}\left(\bar{K}_{p} x_{p}+\sum_{\tau=1}^{t} Q n_{\text {stp }}\right)\left(1-f s_{s t p}\right) \\
& \forall \boldsymbol{t} \in \boldsymbol{T} \boldsymbol{p} \in \boldsymbol{P} \\
& \sum_{l \in L} Q t_{s t i l} \leq\left(\bar{K}_{i} x_{i}+\sum_{\substack{\tau=1 \\
\forall \boldsymbol{t} \in \boldsymbol{T} \boldsymbol{i} \in \boldsymbol{I}}}^{t} Q n_{s t i}\right)\left(1-f s_{s t i}\right) \\
& \sum_{l \in L} Q t_{s t i l} \geq M e_{i}\left(\bar{K}_{i} x_{i}+\sum_{\tau=1}^{t} Q n_{s \tau i}\right)\left(1-f s_{s t i}\right) \\
& \forall \boldsymbol{t} \in \boldsymbol{T} i \in I \\
& \sum_{l \in L} Q t_{s t i l}=\sum_{p \in P} Q t_{s t p i} \forall \boldsymbol{t} \in \boldsymbol{T}, \boldsymbol{i} \in \boldsymbol{I} \\
& Q n_{s t p}=0 \quad \forall \boldsymbol{t} \in \boldsymbol{T} \boldsymbol{p} \in \boldsymbol{p} \boldsymbol{d}_{\boldsymbol{s}}^{\boldsymbol{p}} \\
& Q n_{s t i}=0 \quad \forall \boldsymbol{t} \in \boldsymbol{T} \boldsymbol{i} \in \boldsymbol{i} \boldsymbol{d}_{\boldsymbol{s}}^{\boldsymbol{i}} \\
& Q n_{s t p}=0 \quad \forall \boldsymbol{t} \in \boldsymbol{T} \backslash \boldsymbol{t} \boldsymbol{r}_{s}^{t}, \boldsymbol{p} \in \boldsymbol{P} \\
& Q n_{s t i}=0 \quad \forall \boldsymbol{t} \in \boldsymbol{T} \backslash \boldsymbol{t} \boldsymbol{r}_{s}^{t}, \boldsymbol{i} \in \boldsymbol{I} \\
& Q p_{s t l}=0 \forall \boldsymbol{t} \in \boldsymbol{T} \backslash \boldsymbol{t} \boldsymbol{d}_{s}^{t}, \boldsymbol{l} \in \boldsymbol{L} \\
& Q t_{s t p i} \leq \operatorname{maxt}_{t p i} q t_{s t p i} \\
& \forall \boldsymbol{t} \in \boldsymbol{T}, \boldsymbol{p} \in P, \boldsymbol{i} \in \boldsymbol{I} \\
& Q t_{s t p i} \geq \operatorname{mint}_{t p i} q t_{s t p i} \\
& \forall t \in T, p \in P, i \in I \\
& Q t_{\text {stil }} \leq \operatorname{maxt}_{\text {til }} q t_{\text {stil }} \\
& \forall t \in T, i \in I, l \in L \\
& Q t_{\text {stil }} \geq \operatorname{mint}_{\text {til }} q t_{\text {stil }} \\
& \forall t \in T, i \in I, l \in L \\
& Q n_{s t p} \leq \operatorname{maxn}_{t p} q n_{s t p} \\
& \forall t \in T, p \in P \\
& Q n_{s t p} \geq \operatorname{minn}_{t p} q n_{s t p} \\
& \forall t \in T, p \in P \\
& Q n_{s t i} \leq \operatorname{maxn}_{t i} q n_{s t i} \\
& \forall t \in T, i \in I \\
& Q n_{s t i} \geq \operatorname{minn}_{t i} q n_{s t i} \\
& \forall t \in T, i \in I
\end{aligned}
$$




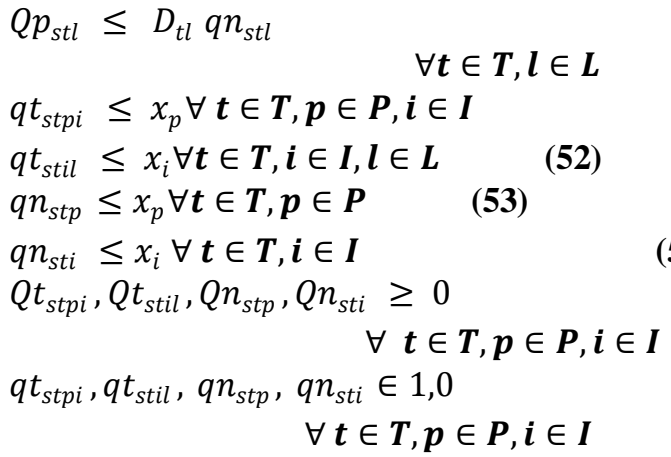

\section{References}

Arabani, A. B., \& Farahani, R. Z. (2012). Facility location dynamics: An overview of classifications and applications. Computers \& Industrial Engineering, 62(1), 408-420.

Badri, H., Bashiri, M., \& Hejazi, T. H. (2013). Integrated strategic and tactical planning in a supply chain network design with a heuristic solution method. Computers \& Operations Research, 40(4), 1

Baymout, M. GLOBAL SUPPLY CHAIN DISRUPTIONS AND MITIGATION STRATEGIES.

Blackhurst*, J., Craighead, C. W., Elkins, D., \& Handfield, R. B. (2005). An empirically derived agenda of critical research issues for managing supply-chain disruptions. International journal of production research, 43(19), 4067-4081.

Cortinhal, M. J., Lopes, M. J., \& Melo, M. T. (2015). Dynamic design and re-design of multi-echelon, multi-product logistics networks with outsourcing opportunities: A computational study. Computers \& Industrial Engineering, 90, 118-131.

Dani, S., \& Ranganathan, R. (2008). Agility and supply chain uncertainty: a scenario planning perspective. International Journal of Agile Systems and Management, 3(3-4), 178-191.

Ghadge, A., Dani, S., \& Kalawsky, R. (2012). Supply chain risk management: present and future scope. The International Journal of Logistics Management, 23(3), 313-339.

Hendricks, K. B., \& Singhal, V. R. (2005). An empirical analysis of the effect of supply chain disruptions on long- run stock price performance and equity risk of the firm. Production and Operations management, 14(1), 35-52.

Hinojosa, Y., Kalcsics, J., Nickel, S., Puerto, J., \& Velten, S. (2008). Dynamic supply chain design with inventory. Computers \& Operations Research, 35(2), 373-391.

Hishamuddin, H., Sarker, R. A., \& Essam, D. (2012). A disruption recovery model for a single stage production-inventory system. European Journal of Operational Research, 222(3), 464-473.
Ivanov, D., Pavlov, A., Dolgui, A., Pavlov, D., \& Sokolov, B. (2016). Disruption-driven supply chain (re)-planning and performance impact assessment with consideration of pro-active and recovery policies. Transportation Research Part E: Logistics and Transportation Review, 90, 7-24.

Klibi, W., \& Martel, A. (2013). The design of robust value-creating supply chain networks. $O R$ spectrum, 35(4), 867-903.

Kristianto, Y., Gunasekaran, A., Helo, P., \& Hao, Y. (2014). A model of resilient supply chain network design: A two-stage programming with fuzzy shortest path. Expert systems with applications, 41(1), 39-49.

Losada, C., Scaparra, M. P., \& O'Hanley, J. R. (2012). Optimizing system resilience: a facility protection model with recovery time. European Journal of Operational Research, 217(3), 519-530.

Mavrotas, G., \& Florios, K. (2013). An improved version of the augmented $\varepsilon$-constraint method (AUGMECON2) for finding the exact pareto set in multi-objective integer programming problems. Applied Mathematics and Computation, 219(18), 9652-9669.

Melo, M. T., Nickel, S., \& Da Gama, F. S. (2006). Dynamic multi-commodity capacitated facility location: a mathematical modeling framework for strategic supply chain planning. Computers \& Operations Research, 33(1), 181-208.

Melo, M. T., Nickel, S., \& Saldanha-da-Gama, F. (2012). A tabu search heuristic for redesigning a multi-echelon supply chain network over a planning horizon. International Journal of Production Economics, 136(1), 218-230.

Peng, P., Snyder, L. V., Lim, A., \& Liu, Z. (2011). Reliable logistics networks design with facility disruptions. Transportation Research Part B: Methodological, 45(8), 1190-1211.

Pimentel, B. S., Mateus, G. R., \& Almeida, F. A. (2013). Stochastic capacity planning and dynamic network design. International Journal of Production Economics, 145(1), 139-149.

Prasanna Venkatesan, S., \& Kumanan, S. (2012). A multi-objective discrete particle swarm optimisation algorithm for supply chain network design. International Journal of Logistics Systems and Management, 11(3), 375-406.

Sahebjamnia, N., Torabi, S. A., \& Mansouri, S. A. (2015). Integrated business continuity and disaster recovery planning: Towards organizational resilience. European Journal of Operational Research, 242(1), 261-273.

Seyedhosseini, S. M., Makui, A., Shahanaghi, K., \& Torkestani, S. S. (2016). Models, solution, methods and their applicability of dynamic location problems (DLPs)(a gap analysis for further research). Journal 
of Industrial Engineering International, 12(3), 311341.

Snyder, L. V., Atan, Z., Peng, P., Rong, Y., Schmitt, A. J., \& Sinsoysal, B. (2016). OR/MS models for supply chain disruptions: A review. IIE Transactions, 48(2), 89-109.

Tang, C. S. (2006). Perspectives in supply chain risk management. International Journal of Production Economics, 103(2), 451-488.

Thanh, P. N., Bostel, N., \& Péton, O. (2008). A dynamic model for facility location in the design of complex supply chains. International Journal of Production Economics, 113(2), 678-693.

Thanh, P. N., Bostel, N., \& Péton, O. (2012). A DC programming heuristic applied to the logistics network design problem. International Journal of Production Economics, 135(1), 94-105.

Thanh, P. N., Péton, O., \& Bostel, N. (2010). A linear relaxation-based heuristic approach for logistics network design. Computers \& Industrial Engineering, 59(4), 964-975.
Torabi, S. A., Baghersad, M., \& Mansouri, S. A. (2015). Resilient facility selection and order allocation under operational and disruption risks. Transportation Research Part E: Logistics and Transportation Review, 79, 22-48.

Wilhelm, W., Han, X., \& Lee, C. (2013). Computational comparison of two formulations for dynamic supply chain reconfiguration with capacity expansion and contraction. Computers \& Operations Research, 40(10), 2340-2356.

Snyder, L. V., \& Daskin, M. S. (2006). Stochastic probust location problems. IIE Transactions, 38(11), 971-985.

SOTO, J. E. T. (2009). Dynamic and robust capacitated facility location in time varying demand environments (Doctoral dissertation, Texas A\&M University). 